\title{
Identification of potential transcriptional regulators of actinorhizal symbioses in Casuarina glauca and Alnus glutinosa
}

Issa Diédhiou ${ }^{1,2,3,4}$, Alexandre Tromas ${ }^{2}$, Maïmouna Cissoko ${ }^{1,2}$, Krystelle Gray ${ }^{1,2}$, Boris Parizot ${ }^{5,6}$, Amandine Crabos ${ }^{2}$, Nicole Alloisio ${ }^{7}$, Pascale Fournier ${ }^{7}$, Lorena Carro ${ }^{7}$, Sergio Svistoonoff ${ }^{1,2,4}$, Hassen Gherbi ${ }^{4}$, Valérie Hocher ${ }^{4}$, Diaga Diouf ${ }^{1,3}$, Laurent Laplaze ${ }^{1,2,4}$ and Antony Champion ${ }^{1,2,4^{*}}$

\begin{abstract}
Background: Trees belonging to the Casuarinaceae and Betulaceae families play an important ecological role and are useful tools in forestry for degraded land rehabilitation and reforestation. These functions are linked to their capacity to establish symbiotic relationships with a nitrogen-fixing soil bacterium of the genus Frankia. However, the molecular mechanisms controlling the establishment of these symbioses are poorly understood. The aim of this work was to identify potential transcription factors involved in the establishment and functioning of actinorhizal symbioses.

Results: We identified 202 putative transcription factors by in silico analysis in 40 families in Casuarina glauca (Casuarinaceae) and 195 in 35 families in Alnus glutinosa (Betulaceae) EST databases. Based on published transcriptome datasets and quantitative PCR analysis, we found that $39 \%$ and $26 \%$ of these transcription factors were regulated during C. glauca and A. glutinosa-Frankia interactions, respectively. Phylogenetic studies confirmed the presence of common key transcription factors such as NSP, NF-YA and ERN-related proteins involved in nodule formation in legumes, which confirm the existence of a common symbiosis signaling pathway in nitrogen-fixing root nodule symbioses. We also identified an actinorhizal-specific transcription factor belonging to the zinc finger C1-2i subfamily we named CgZF1 in C. glauca and AgZF1 in A. glutinosa.

Conclusions: We identified putative nodulation-associated transcription factors with particular emphasis on members of the GRAS, NF-YA, ERF and $\mathrm{C}_{2} \mathrm{H}_{2}$ families. Interestingly, comparison of the non-legume and legume TF with signaling elements from actinorhizal species revealed a new subgroup of nodule-specific $\mathrm{C}_{2} \mathrm{H}_{2}$ TF that could be specifically involved in actinorhizal symbioses. In silico identification, transcript analysis, and phylogeny reconstruction of transcription factor families paves the way for the study of specific molecular regulation of symbiosis in response to Frankia infection.
\end{abstract}

Keywords: Transcription factors, Symbiosis, Actinorhizal plants, Signaling pathway, $\mathrm{C}_{2} \mathrm{H}_{2}$ transcription factors

\footnotetext{
* Correspondence: antony.champion@ird.fr

'Laboratoire Mixte International Adaptation des Plantes et microorganismes

associés aux Stress Environnementaux, Centre de Recherche de Bel Air, BP

1386 CP 18524 Dakar, Sénégal

${ }^{2}$ Laboratoire Commun de Microbiologie IRD/ISRA/UCAD, Centre de

Recherche de Bel Air, Dakar, Sénégal

Full list of author information is available at the end of the article
} 


\section{Background}

Nitrogen is one of the most limiting nutrients for plant growth despite its abundance in the atmosphere. It can only be absorbed by plants as $\mathrm{NH}_{4}{ }^{+}$or $\mathrm{NO}_{3}{ }^{-}$. However, dinitrogen can be reduced by some diazotrophic soil microorganisms, some of which are able to associate with specific plant families. Some of these mutualistic associations lead to the accommodation of bacteria within plant cells in specialized root structures called root nodules. Root nodule symbioses are found in a limited number of plants belonging to the legume, Cannabaceae (Parasponia) and actinorhizal plants. Actinorhizal plants belong to eight angiosperm families that can form symbioses with a filamentous soil bacterium called Frankia. Despite their ecological importance and recent advances in knowledge, the molecular bases of the formation and functioning of actinorhizal symbioses are still poorly understood [1-4]. Recently, transcriptomic analyses in three actinorhizal plants, C. glauca, A. glutinosa and Datisca glomerata $[5,6]$ led to the discovery and characterization of several genes preferentially expressed in actinorhizal nodules following inoculation with Frankia. This global analysis of gene expression revealed that genes of the common symbiotic pathway (SYM) composed of signaling elements required for both root nodule and arbuscular mycorrhizal (AM) symbioses were also conserved in actinorhizal plants $[5,6]$. Furthermore, comparative transcriptome analysis of genes expressed during AM, rhizobial and actinorhizal symbioses suggests the existence of a core set of genes induced in these three endosymbioses [7].

In the last decade, genetic studies on legume symbiosis of the model species (Lotus japonicus and Medicago truncatula) have elucidated the role of transcription factors (TF) at different stages of nodule formation [8]. The first TF described as playing a role in Lotus japonicus nodulation was the NIN (nodule inception) gene for which mutation leads to inhibition of infection and primordia formation [9]. NIN is a transcription factor that is induced during the early stages of nodule organogenesis [10] and is involved in many nodule formation processes [11]. In addition, NIN orthologs have been identified in pea, soybean and $M$. truncatula, where they act downstream of the common SYM genes [12]. Recently, it was demonstrated that in L. japonicus, NIN regulates cortical cell division by targeting two nuclear factors, $L j N F-Y A 1$ and $L j N F-Y B 1$, that are essential for root nodule organogenesis [13]. Furthermore, it was demonstrated that the TF CYCLOPS transactivates NIN expression in a phosphorylationdependent manner leading to root nodule development [14]. NSP1 and NSP2 (nodulation signaling pathway) genes coding for GRAS TF are also specifically involved in the rhizobia-legume symbiosis [15-17], and recent studies suggest their involvement in the arbuscular mycorrhizal symbiosis $[18,19]$. NSP1/NSP2 forms an heterodimer and activates the ERN1 (ethylene response factor) TF required for nodulation by binding to the AT rich region of the promoter, which in turn stimulates the expression of the $E N O D 11$ gene expressed during the pre-infection process [17]. In Medicago truncatula, MtNF-YA1 and MtNF-YA2 also control ENOD11 expression through direct MtERN1 activation [20]. Recently, MtRSD (regulator of symbiosome differentiation), a Cysteine-2/Histidine- $2\left(\mathrm{C}_{2} \mathrm{H}_{2}\right) \mathrm{TF}$, was shown to promote differentiation of bacteria into nitrogen fixing bacteroids [21]. Taken together, these data reveal that specific TF orchestrate plant infection and nodule organogenesis in legumes.

The objective of this study was to identify the TF that regulate the expression of genes involved in the $C$. glauca- /A. glutinosa-Frankia actinorhizal symbioses. Among 14,000 unigenes expressed in roots and nodules of each of the two species, we identified 202 and 195 TF distributed in 40 and 35 families in C. glauca and $A$. glutinosa, respectively. A global analysis of the expression profile of these genes was conducted to identify up- and down-regulated TF encoding genes in nodule versus root, as well as nodule-specific TF. The expression level of several C. glauca and A. glutinosa TF was confirmed by quantitative PCR. Phylogenetic analyses performed in model legumes, species related to actinorhizal plants, and the actinorhizal plants C. glauca and A. glutinosa enabled us to identify ZF1 (zinc finger 1)related transcription factors as potential specific regulators of actinorhizal symbioses.

\section{Results}

\section{Identification of C. glauca and A. glutinosa transcription} factors

To identify transcription factors in actinorhizal plants, tBLASTn searches of the C. glauca and A. glutinosa unigene databases were performed using the DNA-binding domain from the TF database of Arabidopsis thaliana as query sequences. These databases contain 14,327 unigenes for A. glutinosa and 14,868 unigenes for C. glauca [5]. BLAST analysis of the two unigene sets revealed 405 and 358 genes possibly encoding TF in C. glauca and A. glutinosa, respectively. To remove false positives, tBLASTx was performed to check trans-species sequence homologies between Arabidopsis genes and actinorhizal sequences with an e-value cut-off of $1 \mathrm{e}^{-10}$. Using this approach, we narrowed it down to 202 and 195 potential transcription factors distributed in 40 and 35 families in C. glauca and A. glutinosa, respectively (Additional files 1 and 2). No potential members were identified for thirteen families including M-type, E2F/DP, and GeBP in C. glauca and A. glutinosa unigene databases. Each predicted C. glauca and A. glutinosa TF gene was given an arbitrary number. Additional files 1 and 2 list each predicted gene for both species, together with the accession numbers of all unigenes, the 
closest Arabidopsis TF, and detailed BLAST information. Among TF families, the MYB superfamily and the ERF family were the largest, totaling 39 TF for each species. The third largest family was the $\mathrm{C}_{2} \mathrm{H}_{2}$ family, with 18 members in C. glauca and 20 in A. glutinosa, followed by the WRKY, NAC and bHLH families. The remaining families were represented by 1 to 19 members (Table 1 ).

\section{Expression profile of the putative actinorhizal transcription factors}

Microarray data was retrieved from previous published studies [5,7]. First, we used a simple comparative transcriptomics tool called Casuarina Transcriptome Compendium (CTC) to compare the microarray data and generate expression profiles in different conditions. Using CTC, we identified 54 repressed transcription factors and 25 induced in C. glauca nodules compared to non-inoculated roots with a nodule/root fold change $\geq 2$ or $\leq-2$ and a $p$-value $\leq 0.01$ (Figure 1 and Additional file 3 ). The $\mathrm{C}_{2} \mathrm{H}_{2}$ family was the most frequently represented in the up-regulated class followed by the ERF and bHLH families (Additional file 4). Induction of 11 transcription factors in C. glauca nodules was confirmed by quantitative PCR, which also revealed similar induction values than microarray data (Additional file 5). Induction of $C g Z F 1$ was validated by semi-quantitative PCR because its expression was not detectable in the non-inoculated roots needed to calibrate Q-PCR analysis (Additional file 6).

Similarly, we combined available microarray data in A. glutinosa to generate an Alnus Transcriptome Compendium (ATC). This allowed us to identify 30 putative transcription factors induced and 22 others repressed in A. glutinosa nodules compared to non-inoculated roots (Figure 1 and Additional file 7). Similar to the results observed in C. glauca nodules most MYB and WRKY were down-regulated while $\mathrm{C}_{2} \mathrm{H}_{2}$ were induced in nodules, (Additional file 8). Induction in A. glutinosa nodules of seven transcription factors was also confirmed by quantitative PCR (Additional file 5).

\section{Comparison of TF gene expression in AM and actinorhizal symbioses}

This first set of expression data prompted us to investigate TF gene expression during AM symbiosis in $C$. glauca. Mycorrhized roots data were retrieved from a recent publication [7]. Transcriptomic data highlighted 15 downregulated and 7 up-regulated transcription factors in C. glauca mycorrhized roots (Figure 2 and Additional file 9). NAC and GRAS families had the highest number of transcription factors regulated in mycorrhized roots (Additional file 4). Comparing expression in nodules and mycorrhized roots led to the identification of TFs induced during root nodule symbioses but not during AM.
Only CgZF1, CgHD-ZIP2 and CgG2-like5 respectively belonging to $\mathrm{C}_{2} \mathrm{H}_{2}$, HD-ZIP and G2-like families were significantly induced in response to infection by Frankia and not induced in response to AM fungi (Figures 1 and 2). Even though root nodule symbioses are hypothesized to originate from AM, comparative analyses showed that only one transcription factor named CgERF8 (Nod up/ Myc up) was induced both in nodules and mycorrhized roots of C. glauca. Interestingly, CgERF8 was identified as being related to ERN genes from $M$. truncatula known to be induced by Rhizobium and AM [5].

\section{GRAS, NF-YA and ERF transcription factor families in actinorhizal species}

In legumes, several TFs that play key roles in the transduction of bacterial Nod factors have been identified. These include in particular members of the GRAS, NF-YA and ERF families (i.e. NSP1, NSP2, NF-YA1, NF-YA2, ERN1, ERN2 and ERN3) $[15,20,22]$. Therefore we first focused on these families. We screened extra databases using the tBLASTn algorithm in order to identify GRAS, NF-YA and ERF TFs in a third actinorhizal species, D. glomerata [6], relatives of actinorhizal plants in the Rosales and Cucurbitales orders, in legumes (http://www.phytozome.net/search. php?show=blast\&method=Node_rosales-cucurbitales) and in Arabidopsis (TAIR).

The data was used to construct a phylogenetic tree of the GRAS protein family based on the alignment of the complete protein sequences using the maximum likelihood method. Eleven complete $C$. glauca sequences and eight $A$. glutinosa were collected from respective datasets. Based on the phylogenetic analyses, four distinct subfamilies were defined: SCARECROW-like (SCL), SCARECROW (SCR), SHORTROOT (SHR) and DELLA. The tree topology resembled the one found both in rice and Arabidopsis [23,24]. No sequence from C. glauca was close to NSP1 and NSP2. The same result was found for Rosales and Cucurbitales. However, two sequences from A. glutinosa and D. glomerata named AgGRAS7 (AGJ07f_002_D03) and DgNSP1 respectively were identified in the NSP1 group (Figure 3).

Plant genes belonging to the NF-YA family encode putative TFs that are variable in length [25]. Their DNA binding domain shares some similarity with the CCT domain of the nuclear flowering time regulator CONSTANS (CO) [26,27]. NF-YA proteins are characterized by two domains (protein binding and DNA binding domains) that are strongly conserved in all higher eukaryotes examined to date [25]. To clarify the phylogenetic situation of the NF-YA genes identified in the transcriptomes, we built a tree using the maximum likelihood method using only the NF-YA domains. Five of the originally collected sequences had incomplete DNA and protein domains in the C. glauca and A. glutinosa datasets and 
Table 1 Classification of putative transcription factor of Casuarina glauca and Alnus glutinosa into families

\begin{tabular}{|c|c|c|c|c|}
\hline TF family & Casuarina glauca & Alnus glutinosa & Total TF & Domain description \\
\hline AP2 & 3 & 2 & 5 & AP2 domain \\
\hline ARF & 6 & 4 & 10 & Auxin response factor \\
\hline ARR-B & 1 & & 1 & Response regulator contain MYB-like DNA binding domain ARRM (type B) \\
\hline B3 & 1 & & 1 & AP2 like transcriptional factor \\
\hline $\mathrm{BBR} / \mathrm{BPC}$ & 1 & & 1 & Basic pentacysteine \\
\hline BES1 & 1 & & 1 & BRI1-EMS-SUPPRESSOR 1 \\
\hline bHLH & 8 & 15 & 23 & The basic/helix-loop-helix proteins \\
\hline bZIP & 8 & 10 & 18 & Basic Leu zipper (bZIP) TF \\
\hline $\mathrm{C} 2 \mathrm{H} 2$ & 18 & 20 & 38 & Zinc finger, $\mathrm{C} 2 \mathrm{H} 2$ type \\
\hline $\mathrm{C} 3 \mathrm{H}$ & 2 & 3 & 5 & Zn-finger, C-x8-C-x5-C-x3-H type \\
\hline CAMTA & 1 & & 1 & Calmodulin binding transcription factors \\
\hline Co-like & 2 & 2 & 4 & CONSTANS TF,defined by zinc finger N-terminal and CCT domain C-terminal \\
\hline DBB & 4 & 3 & 7 & Double B-box zinc finger \\
\hline Dof & 5 & 1 & 6 & DNA binding with one finger \\
\hline EIL & 1 & 2 & 3 & Ethylene insensitive 3 \\
\hline ERF & 15 & 24 & 39 & Ethylene response factor \\
\hline FAR1 & 2 & 1 & 3 & Far-red impaired responsive directly active transcription of FHY1 and FHL \\
\hline G2-like & 7 & & 7 & Homeodomain-like, GLK proteins belonging GARP superfamily \\
\hline GATA & 1 & 3 & 4 & Zinc-finger Animal contain two C-x2-Cx17-C-x2-C domains type-IV \\
\hline GRAS & 11 & 8 & 19 & SCARECROW (SCR), SHORTROOT (SHR) and DELLA domains \\
\hline GRF & & 1 & 1 & GROWTH-REGULATING FACTOR \\
\hline HB-other & 1 & 1 & 2 & Homeobox domain \\
\hline HD-ZIP & 3 & 6 & 9 & HD-ZIP protein, $\mathrm{N}$ terminus \\
\hline HSF & 1 & 4 & 5 & Heat stress transcription factors, DNA binding C-terminal domains \\
\hline LBD & & 1 & 1 & LOB domain \\
\hline LSD & 2 & 3 & 5 & zinc finger domains, $C x x C \times R \times x L M Y x x G A S x V x C x x C$ type \\
\hline MIKC & 14 & 5 & 19 & MADS-box, MIKC type \\
\hline MYB & 20 & 19 & 39 & MYB DNA-binding domain \\
\hline MYB-related & 3 & 3 & 6 & $\mathrm{~N}$-terminal myb-domain \\
\hline NAC & 14 & 15 & 29 & $\begin{array}{l}\text { No apical meristem (NAM), N-terminal DNA-binding domain and a C-terminal } \\
\text { domain }\end{array}$ \\
\hline NF-YA & 2 & 4 & 6 & $\begin{array}{l}\text { subunit NF-YA, Gln(Q)- and Ser/Thr(S/T)-rich NH2 termini, and a DNA-binding } \\
\text { domain }\end{array}$ \\
\hline NF-YB & 1 & 4 & 5 & NF-Y TF, subunit NF-YB related H2B histones, DNA binding domain \\
\hline NF-YC & 4 & 5 & 9 & NF-Y TF, subunit NF-YC related H2A histones, DNA binding domain \\
\hline Nin-like & 2 & & 2 & Plant regulator RWP-RK \\
\hline RAV & & 1 & 1 & B3 domain, DNA binding domain and single AP2/ERF domain \\
\hline S1Fa-like & 1 & 1 & 2 & S1F binding site, NLS and a putative DNA binding helix \\
\hline SBP & 1 & & 1 & Two zinc-binding sites, Cys3HisCys2HisCys or Cys6HisCys sequence motif \\
\hline SRS & & 1 & 1 & Zn-finger, LRP1 type \\
\hline TALE & 5 & 3 & 8 & Homeodomain \\
\hline TCP & 2 & 2 & 4 & TCP TF \\
\hline Trihelix & 1 & 2 & 3 & DNA-binding domain, GT factor \\
\hline WOX & 1 & & 1 & Homeobox domain, WOX class \\
\hline
\end{tabular}


Table 1 Classification of putative transcription factor of Casuarina glauca and Alnus glutinosa into families (Continued)

\begin{tabular}{lrrrl}
\hline WRKY & 15 & 15 & 30 & DNA-binding WRKY \\
Whirly & & 1 & 1 & DNA-binding, Defense response \\
ZF-HD & 1 & 1 & Homeobox domain, ZF-HD class \\
Total TF & $\mathbf{2 0 2}$ & $\mathbf{1 9 5}$ & $\mathbf{3 9 7}$ & \\
Total families & $\mathbf{4 0}$ & $\mathbf{3 5}$ & $\mathbf{4 5}$ & \\
\hline
\end{tabular}

$\mathrm{TF}=$ Transcription factor.

were excluded from further analysis. The NF-YA tree includes 6 major groups (Groups I-VI) also found in legumes (Figure 4, [25]). Interestingly, Group I containing MtNF-YA1 and MtNF-YA2 from M. truncatula includes $C g N F-Y A 1$ from C. glauca. In the present study, Dgcomp3430_c0_seq7 from D.glomerata is in Group II. Several genes belonging to Group II show a strong expression mainly in seeds $[26,28]$.

To study the phylogenetic relationships between the ERF genes in the actinorhizal species, multiple alignment analysis was performed using amino acid sequences in the AP2/ERF domain. Ten of the originally collected sequences had incomplete AP2 domains in the C. glauca and A. glutinosa datasets and were excluded from further analysis. The ERF tree distinguished the 10 major groups (Groups I-X) also found in rice, cotton, and Arabidopsis (Figure 5, [29,30]). At least one C. glauca and A. glutinosa ERF TF was found in the main groups except for Group VI. Group VII, which included five ERFs from Arabidopsis, was the most represented by the actinorhizal ERF transcription factor with three sequences in C. glauca and five sequences in A. glutinosa. However, several changes in the groups were observed (Figure 5). Group IX was divided into three subgroups [29]. In the present study, the phylogenetic tree showed that subgroup IXb was no longer related to subgroup IXa and IXc. Group IX contained five sequences from A. glutinosa and two sequences from the model legume Lotus japonicus including LjERF1. $L j E R F 1$ functions as a positive regulator of nodulation [31]. AgERF18 is the sequence most closely related to LjERF1. Group V was divided into two subgroups [29]. In the present study, the phylogenetic tree showed subgroups $\mathrm{Va}$ and $\mathrm{Vb}$ grouped with Group VIIIa. Interestingly, Group Vb which contained ERN1, ERN2 and ERN3 from M. truncatula, also includes two ERFs, CgERF8 and Dgcomp6569, from C. glauca and D. glomerata, respectively. The reliability of Group V clustering was supported by the presence of common motifs outside the AP2/ERF domain. CgERF8 and Dgcomp6569 as well as ERNs possess the CMV-3 or CMV-4 motifs, like in rice and Arabidopsis (data not shown) [29].

\section{$\mathrm{C}_{2} \mathrm{H}_{2}$ transcription factors in actinorhizal symbiosis}

From the global analysis, the large number of its members and their regulation during actinorhizal symbioses make the $\mathrm{C}_{2} \mathrm{H}_{2}$ family particularly interesting. We therefore

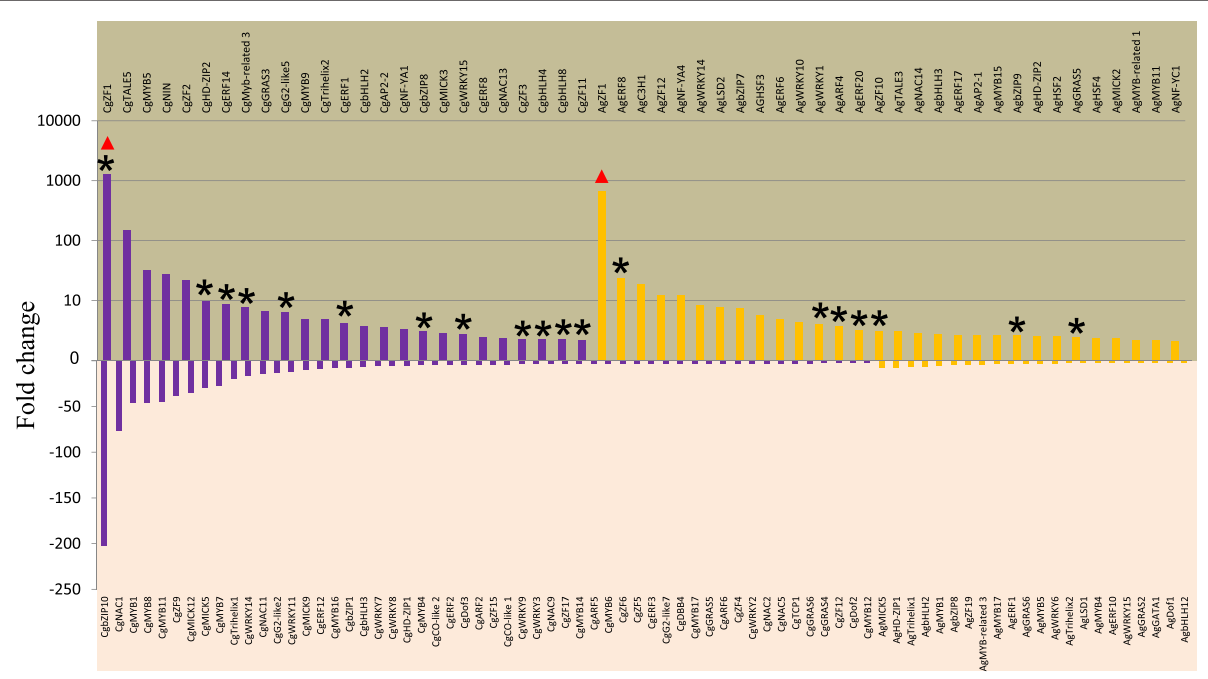

Figure 1 Expression profiles of potential transcription factors in Casuarina glauca and Alnus glutinosa nodules compared to non-inoculated roots. Purple and orange bars represent putative transcriptional regulators from C. glauca and A. glutinosa respectively. Bars represent fold change in nodules compared to non-inoculated roots (Fold change or FC $\geq 2$ or $\leq-2$ and $p$-value $<0.01$ ). Transcription factors induced and repressed in C. glauca and A. glutinosa nodules are highlighted in tanned brown and light pink respectively. Astericks identify the genes confirmed by Q-PCR. Red triangles identify the transcription factors (CgZF1 and AgZF1) most strongly induced in the nodules. 


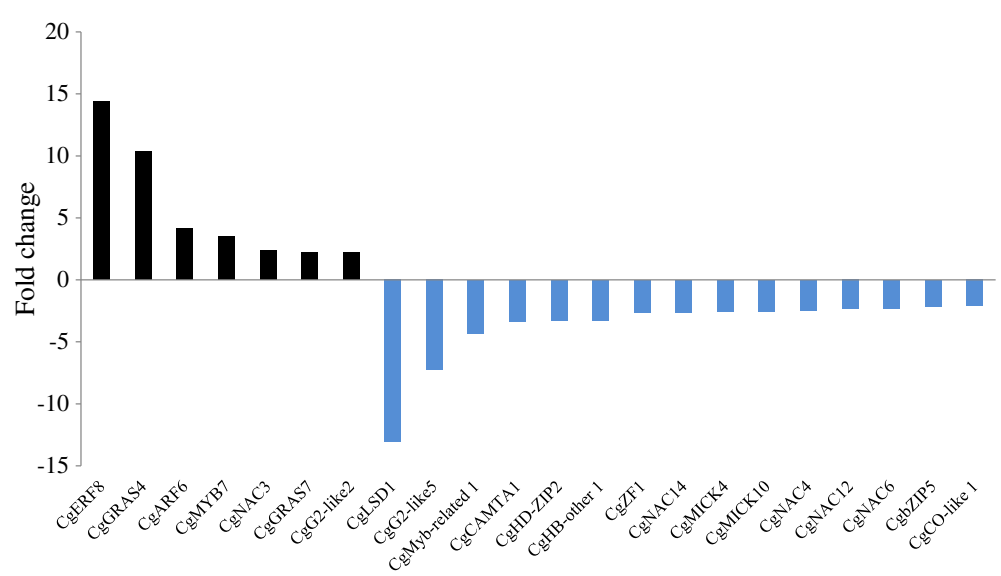

Figure 2 Expression profiles of potential transcription factors in C. glauca mycorrhizal roots compared to non-inoculated roots. Bars represent fold change in mycorrhizal roots compared to non-inoculated roots (Fold change or FC $\geq 2$ or $\leq-2$ and $p$-value $<0.01$ ). Black and blue bars represent up and down-regulated transcriptional regulators in C.glauca mycorrhizal roots. Transcription factors induced and repressed in C. glauca mycorrhizal roots are highlighted in tanned brown and light pink respectively.

decided to study this family further, and in particular two of its members, $C g Z F 1$ and $A g Z F 1$. Expression data showed that four transcription factors of this family were induced and seven less expressed in C. glauca nodules than in non-inoculated roots (Figure 1; Additional file 4).
CgZF1 was specifically induced during nodulation and no $\mathrm{C}_{2} \mathrm{H}_{2}$ were induced in C. glauca mycorrhized roots (Figures 1 and 2 and Additional files 3 and 4). Analysis of the A. glutinosa nodule transcriptome showed that one $\mathrm{C}_{2} \mathrm{H}_{2}$ was down-regulated and three were up-regulated in

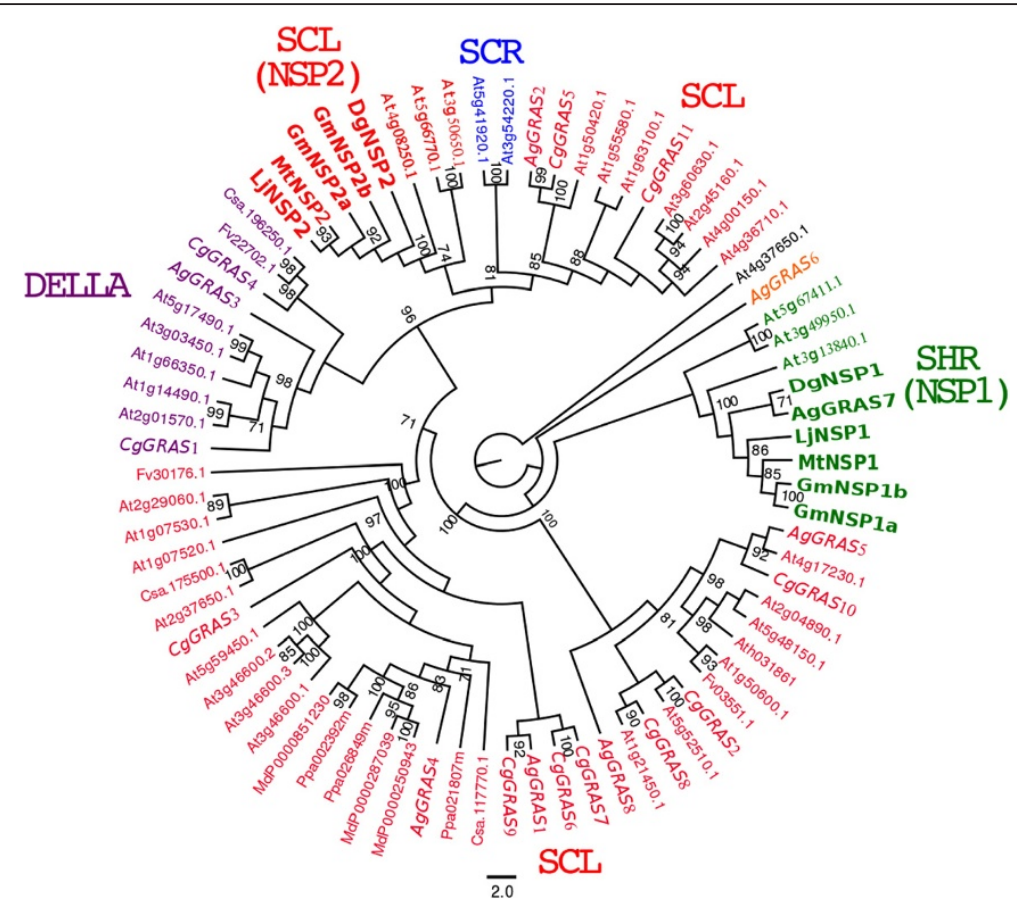

Figure 3 Phylogenetic tree of the GRAS protein family. Three GRAS proteins from C. sativus, P. persica, M. domestica and $F$. vesca each were retrieved using a BLAST-P search performed in phytozome and two sequences DgNSP1 (comp755_c1_seq1) and DgNSP2 (comp1841_c1_seq1) of D. glomerata (https://fido.nsc.liu.se/) [6]. We also include 36 sequences available in the genomes of A. thaliana, 2 from M. truncatula, 2 from L. japonicus, 4 from G.max, 11 from C. glauca and 8 from A. glutinosa. In italic, transcription factors C.glauca and A.glutinosa. The GRAS family comprises 4 subfamilies referred to as SCL, SCR, SHR and DELLA. NSP1 and 2 belong to SHR and SCL subfamilies respectively. AgGRAS7 is closely related to DgNSP1 (green fat letters). No sequence from C. glauca is present in the NSP1 and NSP 2 groups (red fat letters). The tree was rooted with A. thaliana sequence AT3G37650.1. One hundred bootstrap replications were used to evaluate statistical support for branches. Branches with less than $70 \%$ bootstrap support were collapsed. 


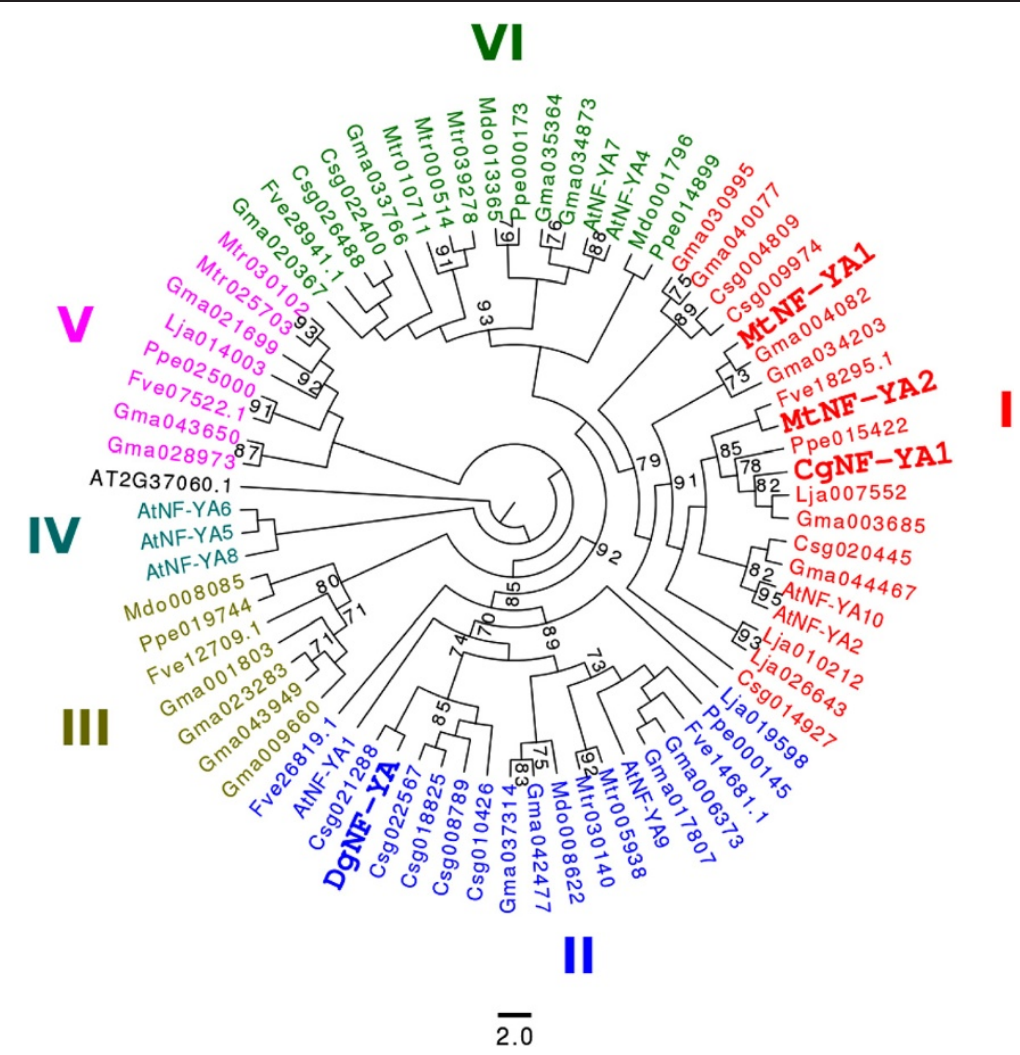

Figure 4 Phylogenetic tree of the NF-YA protein family. We include NF-YA proteins from A. thaliana (9 proteins), M. truncatula (9), L. japonicus (5) and G. max (21). Sequences of C. sativus (11), P. persica (6), M. domestica (4) and F. vesca (6) were retrieved using a BLAST-P search performed in phytozome and one sequence of D. glomerata (https://fido.nsc.liu.se/) [6]. In the present study, NF-YA tree comprises six groups: I to VI. CgNF-YA1 belongs to Group I. DgNF-YA (comp3430_C0_seq7) is in Group II. The tree was rooted with A. thaliana sequence AT3G37650.1 (NF-YB). One hundred bootstrap replications were used to evaluate statistical support for branches. Branches with less than $70 \%$ bootstrap support were collapsed.

nodules (Figure 1; Additional file 8). Remarkably, like in the C. glauca TF gene expression dataset, a $\mathrm{C}_{2} \mathrm{H}_{2} \mathrm{TF}$, $A g Z F 1$, showed the strongest gene induction in A. glutinosa nodules as indicated by the red triangles in Figure 1. The $\mathrm{C}_{2} \mathrm{H}_{2}$ family is characterized by the presence of a "zinc finger" DNA binding domain containing one or two "QALGGH" conserved motif(s) [32]. CgZF1 contains two QALGGH motifs at positions 85-105 and 142-162. Furthermore, a putative EAR (ethylene-responsive element-binding associated amphiphilic repression) repressor domain with the signature (S)/(F)DLN(L)/(F)XP was identified at the C-terminal positions 176 and 180 of CgZF1. The "EAR domain" is known to be responsible for the repression of gene expression [33,34]. The presence of this domain in $\mathrm{C}_{2} \mathrm{H}_{2} \mathrm{C} 1-2 \mathrm{i}$ proteins suggests that they are transcriptional repressors of their target genes. Finally, a nuclear localization signal (NLS) involved in the translocation of protein to the nucleus was also identified at position 31-36 in its N-terminal end. Alignment of AgZF1, with the C. glauca CgZF1 revealed the position of DNA-binding including two "QALGGH" conserved motifs, an EAR domain and a NLS within the protein sequence and a high degree of identity (79\%) with CgZF1 (Additional file 10).

To further characterize the relationship between CgZF1 and AgZF1, a phylogenetic tree based on the alignment of the complete protein sequences was generated (Figure 6). The sequence of $M$. sativa Mszpt2-1 was added to the dataset because it encodes a $\mathrm{C}_{2} \mathrm{H}_{2}$ TF and plays a role in legume symbiosis [35]. The phylogenetic tree distinguished two major groups, Groups I and II. Group I did not contain C. glauca, A. glutinosa and A. thaliana $\mathrm{C}_{2} \mathrm{H}_{2}$ TF. Group II was divided into three subgroups named A, B and C. Subgroups II-B and II-C contained seven and five $\mathrm{C}_{2} \mathrm{H}_{2}$ TFs from actinorhizal plants, respectively. Interestingly, one cluster in subgroup II-B containing CgZF1 and $A g Z F 1$ was only made up of sequences from Rosales, Fagales, and Cucurbitales and did not contain any legume sequence. Our analysis also confirmed that CgZF1 and AgZF1 protein sequences are very close and are most probably orthologs (79\% similarity, data not shown; bootstrap value, 95\%, Figure 6). This suggests that ZF1-like transcription factors evolved after the divergence between Fabales and the Rosales/Cucurbitales/Fagales clade and the 


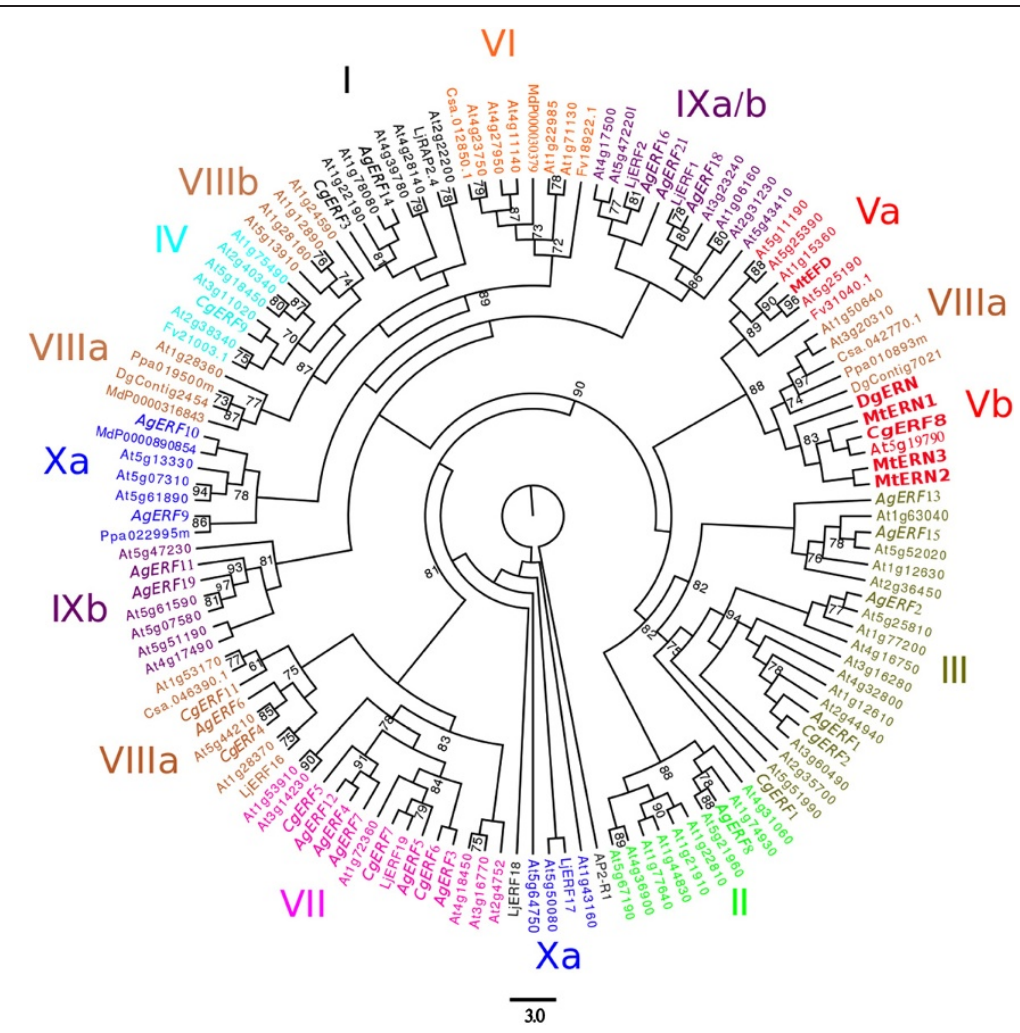

Figure 5 Phylogenetic tree of the ethylene-response factor (ERF) protein family. Three ERF proteins from C. sativus, P. persica, M. domestica and $F$. vesca each were retrieved using a BLAST-P search performed in phytozome and 2 sequences DgERN (comp6569_c0_seq1) and DgContig2454 (Contig2454) from D. glomerata (https://fido.nsc.liu.se/) [6]. We also include 74 ERF from A. thaliana, 4 from M. truncatula and 7 from L. japonicus. In italic, transcription factors C.glauca and A.glutinosa. The ERF family comprises ten groups referred as I to X. CgERF8 and DgERN are in Group V (red fat letters). They are closer to MtERN1 than MtERN2 and MtERN3. The tree was rooted to APETALA2 AP2-domain R1 (At4g36920). One hundred bootstrap replications were used to evaluate statistical support for branches. Branches with less than $70 \%$ bootstrap support were collapsed.

common ancestor of actinorhizal Fagales recruited these genes for functions related to nodulation.

\section{Discussion}

The search for putative transcription factors is an important first step in characterizing the signaling pathways that control the establishment and functioning of actinorhizal symbioses. This analysis enabled us to identify 202 and 195 transcription factors in C. glauca and A. glutinosa, respectively. The use of transcriptome data generated in C. glauca showed that half the putative transcription factors are regulated in nodules and mycorrhizae (Additional file 11). In A. glutinosa, $26 \%$ are significantly regulated in nodules. In addition, the transcriptome data pointed to co-regulation of $C g Z F 1$ and $A g Z F 1$, which belong to the $\mathrm{C}_{2} \mathrm{H}_{2}$ family.

In silico studies have already been conducted using several plant models to optimize automated annotation of transcription factors and to obtain a plant TF atlas. For example, 2,023 transcription factors have been identified in A. thaliana, 1,613 in M. truncatula, 1,275 in $L$. japonicus and 3,557 in G. max [36]. These transcription factors are characterized by their DNA binding domains, which are specific to each family. This led to the identification of 58 families in A. thaliana, 56 in M. truncatula and L. japonicus and 57 in G. $\max$ [36]. The putative transcription factors are distributed in 40 families in C. glauca and 35 in A. glutinosa. The difference between these and other plant models can be explained by the fact that $C$. glauca and A. glutinosa genomic data come from 14,327 unigenes for A. glutinosa and 14,868 unigenes for $C$. glauca. As part of the effort to better understand the biology of actinorhizal symbiosis, large-scale cDNA sequencing using next generation sequencing technologies will provide a means to identify new TF genes and to produce an actinorhizal gene expression atlas.

Among the transcription factors identified, CgNIN (Casuarina glauca nodule inception) showed high similarity with MtNIN (Medicago truncatula nodule inception) and LjNIN (Lotus japonicus nodule inception). CgNIN was strongly induced in nodules (fold change $=27$ ) and not significantly induced in mycorrhized roots (Figures 1 and 2). We also identified another gene called CgERF8 coding for an ERF transcription factor. This gene was 


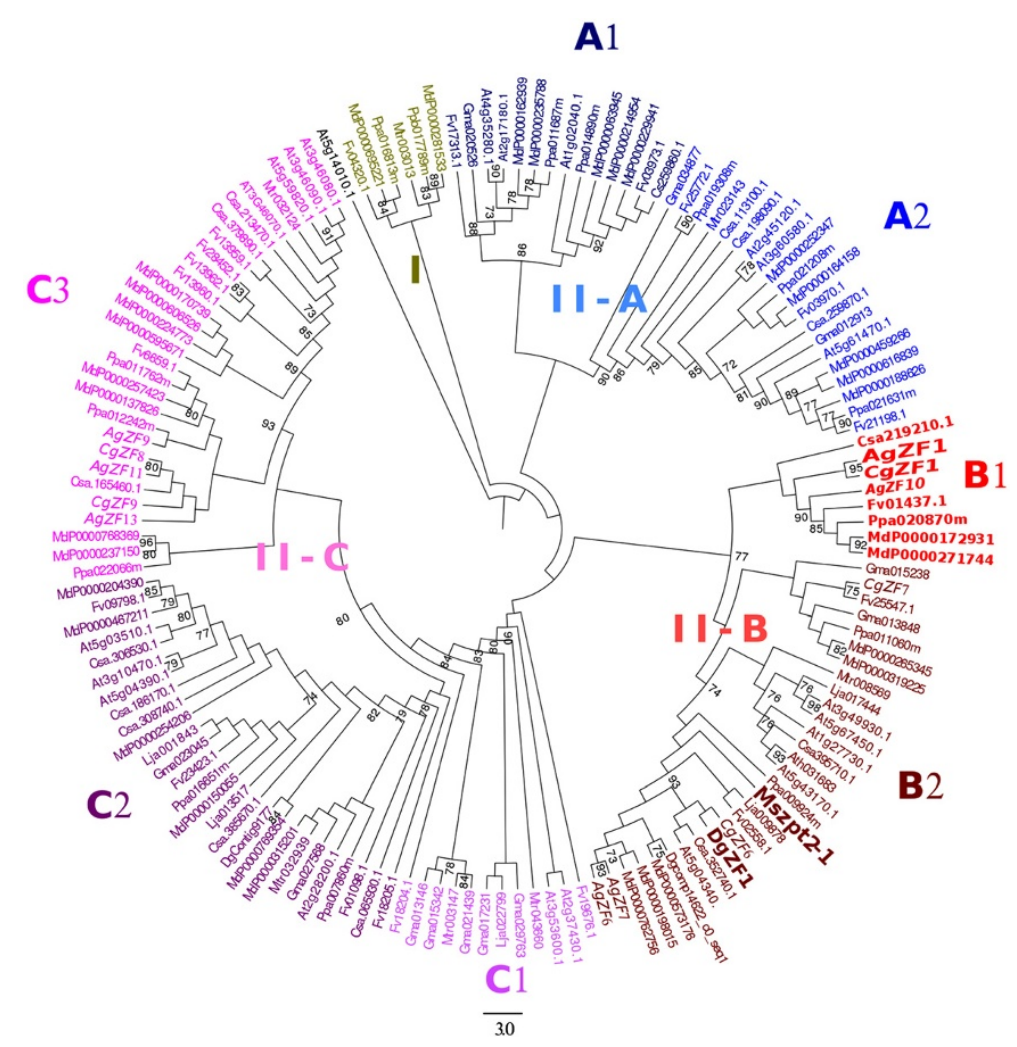

Figure 6 Phylogenetic tree of the $\mathrm{C}_{2} \mathrm{H}_{2}$ C1-2i protein family. A maximum likelihood tree representing relationships among $24 \mathrm{C}_{2} \mathrm{H}_{2}$ proteins from A. thaliana, 13 from G.max, 7 from M. truncatula and A. glutinosa, 5 from L. japonicus and C. glauca, 3 sequences DgZF1 (Contig7810), Dgcomp14622_c0_seq1 (comp14622_c0_seq1) and DgContig9177 (Contig9177) from D. glomerata. The tree was rooted with A. thaliana sequence AT5G14010.1 (C1-1i). In italic, transcription factors C.glauca and A.glutinosa. The $\mathrm{C}_{2} \mathrm{H}_{2} \mathrm{C} 1-2 \mathrm{i}$ family comprises two groups referred to as I and II. Group II is divided into three subgroups named A, B and C. The subgroups are also divided into several clusters. Cluster II-B1 contains sequences of actinorhizal plants and related sequences from Rosales and Cucurbitales include CgZF1 and AgZF1 (red fat writing). The sequence of $M$. sativa Mszpt2-1 was added to the dataset because it encodes a $\mathrm{C}_{2} \mathrm{H}_{2}$ C1-2i type transcription factor. The nodes represent bootstrap values $(\geq 70 \%)$ from 100 replications.

induced in both C. glauca nodules and mycorrhizae. In M. truncatula, a gene encoding a transcription factor belonging to the ERF family named MtERN2 [37] presents the same expression profile as CgERF8. These two genes share $55 \%$ similarity and conservation of specific domains (data not shown). In addition, we identified $C g N F-Y A 1$, a close homologue of MtNF-YA1 and MtNF$Y A 2$, two TFs involved in the activation of MtERN1 [20]. CgNF-YA1 is induced in actinorhizal nodules (fold change $=3,296$, see Figure 1). No sequence of $C$. glauca belongs to the NSP1 and NSP2 groups. In A. glutinosa, AgGRAS7 belongs to the NSP1 group and is closely related to DgNSP1 (bootstrap value $=71 \%$ ). Consequently, we suggest that CgNIN, AgNSP1, CgNF-YA1 and CgERF8 could be functional homologs of NIN, NSP1, NF-YA and $E R N 1$ in legumes. Functional complementation of legume mutants by the potential actinorhizal orthologous genes could be used to test this hypothesis.

Analysis of the expression of the putative transcription factors was performed using transcriptome data to investigate their expression during the C. glauca-/A. glutinosa-Frankia interactions. This investigation revealed 25 induced TFs in C. glauca or A. glutinosa nodules. Recently, 192 genes encoding putative transcription factors strongly induced in nodules were identified in M. truncatula [38]. These TFs were classified in eight functional groups of genes differentially regulated in $M$. truncatula nodules in seven experimental conditions after inoculation with the wild type and three Nod- mutant strains of Sinorhizobium meliloti. Transcription factors belonging mainly to the $\mathrm{C}_{2} \mathrm{H}_{2}$, MYB, WRKY and ERF families appeared to be nodule-specific, whereas members of the ERF and WRKY families were over-represented in the TF dataset [38]. Similarly, transcriptome analysis of $L$. japonicus led to the identification of $20 \mathrm{TF}$ that expression was up-regulated during nodulation showing that members of the ERF family are the most abundant TF [31]. Remarkably, members of the ERF and WRKY families of TF were also abundant in our study, and expression analysis showed that nine of them were induced in actinorhizal nodules. ERF 
and WRKY TFs are often involved in defense responses to abiotic and biotic stresses [39-41]. As hypothesized for legume and rhizobium symbioses, ERF and WRKY TFs could be involved in the regulation of downstream defense responses to inhibit excess Frankia infection under nitrogen-sufficient conditions.

Three genes named CgZF1, CgHD-ZIP2 and CgG2-like5 belonging to the $\mathrm{C}_{2} \mathrm{H}_{2}$, HD-ZIP and G2-like families, respectively, are specific to the C. glauca-Frankia interaction. A TF named $A g Z F 1$ exhibited the same strong induction in $A$. glutinosa nodules as CgZF1. In addition, these two genes were also seen to have the same protein structure and phylogenetic analysis indicate they are most likely orthologous. In legumes, two $\mathrm{C}_{2} \mathrm{H}_{2}$ TFs are involved in nodule functioning. In $M$. truncatula, MtRSD represses transcription of the secretory pathway gene, VAMP721a, required for symbiosome formation, suggesting that MtRSD is directly involved in symbiosome and bacteroid differentiation [21]. MtRSD is a $\mathrm{C}_{2} \mathrm{H}_{2}$ TF but is phylogenetically distant from CgZF1 and AgZF1 (data not shown). In M. sativa, a gene called Mszpt2-1 is involved in the differentiation of nitrogen-fixing cells [35]. Mszpt2-1 possesses two "QALGGH" motifs and an EAR motif as well as CgZF1 and AgZF1. Mszpt2-1, CgZF1 and AgZF1 belong to the same $\mathrm{C}_{2} \mathrm{H}_{2}$ Group II (Figure 6). Functional complementation studies will determine whether $C g Z F 1$ and $A g Z F 1$ are functionally equivalent to putative orthologous genes identified in legumes. Recent studies showed that repressors containing the EAR motif play a role in defense responses to plant biotic and abiotic stress [42]. CgZF1 and AgZF1 could repress the expression of defense genes of actinorhizal plant in response to signals from Frankia to allow colonization of new cells in nodules. Finally, CgZF1 and $A g Z F 1$ could be used as functional markers to investigate the regulation of actinorhizal symbiosis interaction.

\section{Conclusions}

The in silico identification and transcriptome analysis of TFs in C. glauca and in A. glutinosa that we carried out represents a crucial step forward in the elucidation of the molecular events underlying actinorhizal and mycorrhizal symbioses. Coupled with a phylogenetic study, these analyses enabled identification of a nodulation specific gene in C. glauca named CgZF1 and its ortholog in $A$. glutinosa named AgZF1. These two new genes are most probably negative regulators which may play a crucial role in actinorhizal symbiosis. The development of efficient techniques to transform C. glauca [43] now allows us to perform functional studies of $C g Z F 1$.

\section{Availability of supporting data}

EST sequences reported in this paper have been deposited in the GenBank (accession nos. CO036851-CO0388878) and EMBL (accession nos. FQ312199-FQ377516) databases. The normalized and raw microarray data values have been deposited in the Gene Expression Omnibus database (www.ncbi.nlm.nih.gov/geo; accession nos. GPL10929 and GSE24153 for C. glauca and A. glutinosa respectively).

In addition, all nucleic acid sequences used in this study have been included in Additional files 1 and 2. Proteins sequences used to produce phylogenetic data sets have been added in Additional file 12.

\section{Methods}

\section{Database search}

Fifty-eight predicted DNA-binding domain protein sequences from Arabidopsis were used as query sequences for tBLASTn searches against the predicted C. glauca and A. glutinosa proteins in a previously described database (http://www.ncbi.nlm.nih.gov/nucest/; Accession numbers CO036851 to CO0388878 and FQ312199 to FQ377516) [5]. Arabidopsis DNA-binding domain sequences were retrieved from a multi-alignment available in DATF (Database Arabidopsis Transcription Factors: http:// planttfdb.cbi.edu.cn/ version 2.0). The TAIR database (http://www.arabidopsis.org/Blast/) was used to confirm the affiliation of each putative transcription factor to one of the 58 families. tBLASTx were carried out to check trans-species sequence homologies between genes with an e-value cut-off of $1 \mathrm{e}^{-10}$ and genes were annotated according to the TAIR database.

\section{Expression data of putative TF in actinorhizal nodules and mycorrhizal roots}

The putative TF expressed in C. glauca (Accession number GPL10929) and in A. glutinosa (Accession number GSE24153) were retrieved from microarray data [5,7]. A transcription factor was considered differentially expressed if its fold change was greater than or equal to 2 and its p-value lower than or equal to 0.01 .

For quantitative RT-PCR analyses, total RNA was purified from roots and nodules by ultracentrifugation [44] for C. glauca and using the RNeasy plant mini kit (Qiagen, Courtaboeuf, France, see [45]) for A. glutinosa. RNA was quantified using a Nanodrop (Thermo Fisher Scientific, Courtaboeuf, France) and analyzed using a Bioanalyzer 2100 according to the manufacturer's instructions (Agilent, Santa Clara, CA, USA). For C. glauca analyses, reverse transcription were performed on 0.5-1 $\mu \mathrm{g}$ of RNA using the SuperScript ${ }^{\circ}$ reverse transcriptase III kit (Invitrogen Life Science, Carlsbad, CA, USA). For A. glutinosa analyses, reverse transcription was performed with $9 \mu \mathrm{g}$ of RNA using Transcriptor Revers transcriptase and oligo $(\mathrm{dT})_{15}$ primer (Roche, Mannheim, Germany). Reverse transcription (RT) and real time quantitative PCR (qRTPCR) were performed with the same three biological replicates of nodules and non-inoculated roots. Primers 
were designed using Beacon designer software (Premier Biosoft International, Palo Alto, CA, USA). Quantitative RT-PCR analyses were conducted as previously described [5] using primers listed in supporting information (Additional file 13). For C. glauca, amplifications were performed using a Stratagene Mx3005P thermal cycler, (Agilent, Palo Alto, CA, USA) with the Brilliant II SYBR Green QPCR Master Mix (Agilent, Palo Alto, CA, USA) programmed for a pre-denaturation step of $5 \mathrm{~min}$ at $95^{\circ} \mathrm{C}$ followed by 40 cycles of $10 \mathrm{~s}$ at $95^{\circ} \mathrm{C}$ and $30 \mathrm{~s}$ at $60^{\circ} \mathrm{C}$ and $72^{\circ} \mathrm{C}$ for $15 \mathrm{~s}$. For A. glutinosa, amplification was run on a LightCycler 480 (Roche) using LightCycler 480 SYBR Green I Master (Roche) under the following conditions: $95^{\circ} \mathrm{C}$ for $5 \mathrm{~min}$; 45 cycles of $95^{\circ} \mathrm{C}$ for $20 \mathrm{~s}, 60^{\circ} \mathrm{C}$ for $20 \mathrm{~s}$ and $72^{\circ} \mathrm{C}$ for $15 \mathrm{~s}$. The $C$. glauca ubiquitin gene (CgUbi) and the A. glutinosa gene (AgUbi) were used as controls as reported in [44] and, respectively [5].

\section{Semi-quantitative PCR analysis}

cDNA from C. glauca uninfected roots, nodules, nonmycorrhizal roots and mycorrhizae were obtained by reverse transcription RNA as described in [5]. CgZF1 was amplified by PCR using specific sense and antisense primers $C g Z F 1$ (Additional file 13). CgUBI was used as control. Amplification was performed using a GeneAmp ${ }^{\circ}$ PCR System 2400 (Perkin Elmer) programmed for a predenaturation step of $2 \mathrm{~min}$ at $94^{\circ} \mathrm{C}$ followed by 32 cycles of $30 \mathrm{~s}$ at $94^{\circ} \mathrm{C}$ and $30 \mathrm{~s}$ at $62^{\circ} \mathrm{C}$ and $72^{\circ} \mathrm{C}$ for $45 \mathrm{~s}$.

\section{Phylogenetic relationship between transcription factors}

Phylogenetic analysis was performed using the maximum likelihood method on protein sequences from the four TF families: $\mathrm{C}_{2} \mathrm{H}_{2}$, ERF, GRAS and NF-YA, identified in $C$. glauca and $A$. glutinosa. We used the default algorithms and settings implemented in www.phylogeny.fr. Briefly, a protein-based alignment of full-length sequences or conserved domains was generated using MUSCLE [46], this alignment was then curated using the Gblocks [47] to remove poorly aligned positions and gaps and a phylogenetic tree was calculated using PhyML [48]. Trees were edited with FigTree ("http://tree.bio.ed.ac.uk/software/ figtree/"). We used AT5G14010.1, AP2-R1, AT4G36710.1 and AT2G37060.1 to root the $\mathrm{C}_{2} \mathrm{H}_{2}$, ERF, GRAS and NF-YA trees respectively. Trees were constructed based on sequence alignments of DNA binding domains for ERF and NF-YA and complete sequences for $\mathrm{C}_{2} \mathrm{H}_{2}$ and GRAS. The DNA binding domains or protein sequences of these four TF families from A. thaliana (http://planttfdb.cbi.edu.cn/index.php?sp=At), M. truncatula (http:// planttfdb_v1.cbi.pku.edu.cn:9010/web/index. $\mathrm{php}$ ?sp=mt), L. japonicus (http://planttfdb_v1.cbi.pku.edu. cn:9010/web/in dex.php? sp=lj), G. $\max$ (http://planttfdb. cbi.edu.cn/index.php?sp=Gma), Cucumis sativus, Prunus persica, Malus domestica and Fragaria vesca (http://www. phytozome.net/search.php?show=blast\&method=Node_ rosales-cucurbitales) were added to the dataset. The bootstrap values represent a percentage of 100 repetitions.

\section{Additional files}

Additional file 1: Potential transcription factors in C. glauca.

Additional file 2: Potential transcription factors of $A$. glutinosa.

Additional file 3: Putative transcription factors up and down-regulated in C. glauca nodules.

Additional file 4: Distribution of transcription factors families regulated in C. glauca nodules and Mycorrhizae.

Additional file 5: Validation of few transcription factors up regulated in C. glauca and $A$. glutinosa nodules by real time quantitative PCR.

Additional file 6: Validation of transcriptome data by RT-PCR. $\mathrm{NR}=$ non-inoculated root, $\mathrm{NOD}=$ Nodule, $\mathrm{NMR}=$ Non-mycorrhizal root and $\mathbf{M R}=$ mycorrhizae. $\mathrm{CgZF1}=\mathrm{C}$. glauca Zinc Finger 1, CgUBl=C. glauca Ubiquitin (Control). Expression data from microarray of CgZF1 were confirmed by semi-quantitative RT-PCR. The expression of CgUB/ is constitutive and standardizes the expression of CgZF1.

Additional file 7: Putative transcription factors up and down-regulated in A. glutinosa nodules.

Additional file 8: Distribution of transcription factors families regulated in A. glutinosa nodules.

Additional file 9: Putative transcription factors up and down-regulated in C. glauca mycorrhizae.

Additional file 10: Multiple sequence alignment of CgZF1 and ZF1-like proteins from Alnus glutinosa, Cucumis sativus, Fragaria vesca, Prunus persica and Malus domestica.

Additional file 11: Percentage of transcription factors regulated in C. glauca nodule and mycorrhizae.

Additional file 12: Protein sequences of the GRAS, NF-YA, ERF and $\mathrm{C}_{2} \mathrm{H}_{2} \mathrm{C} 1-2 \mathrm{i}$ TF used to generate the phylogenic trees.

Additional file 13: Primers list for semi or quantitative PCR.

\section{Abbreviations}

EST: Expressed sequence tag; PCR: Polymerase chain reaction; qRT-PCR: Quantitative real time-polymerase chain reaction; TAIR: The Arabidopsis Information resource; BLAST: Basic local alignment search tool; NCBI: National Center for Biotechnology Information; DATF: The Database of Arabidopsis Transcription factors; CDS: Coding sequence.

Competing interests

The authors declare that they have no competing interests.

\section{Authors' contributions}

$I D, K G, A T$ and $A C$ performed database searches and analyzed data. AC, MC, NA, PF, LC and VH performed QRT-PCR experiments. ID, SS, and AC performed phylogenetic analysis. BP contributed analytical tools. AT, VH, SS, $H G, D D, L L$, and $A C$ contributed ideas and analyzed data. $L L$ and $A C$ initiated the project, and designed the experiments. ID, DD and AC obtained funding. ID, AT and AC wrote the manuscript. All the authors commented on and approved the manuscript prior to submission.

\section{Acknowledgements}

ID and AT were supported by a grant from the IRD Department of CapacityBuilding for Southern Scientific Communities (IRD-DPF). We thank Dr Mame Oureye Sy (UCAD) for access to the qPCR and Dr Katharina Pawlowski who helped us in the search for orthologous sequences for ZF1, ERN, NF-YA, NSP1 and NSP2 in the Datisca glomerata database. 


\section{Author details}

'Laboratoire Mixte International Adaptation des Plantes et microorganismes associés aux Stress Environnementaux, Centre de Recherche de Bel Air, BP 1386 CP 18524 Dakar, Sénégal. ²Laboratoire Commun de Microbiologie IRD/ ISRA/UCAD, Centre de Recherche de Bel Air, Dakar, Sénégal. ${ }^{3}$ Laboratoire Campus de Biotechnologies Végétales, Département de Biologie Végétale, Faculté des Sciences et Techniques, Université Cheikh Anta Diop, Dakar BP 5005Dakar-Fann, Sénégal. ${ }^{4}$ Institut de Recherche pour le Développement (IRD), UMR DIADE, Equipe Rhizogenèse, Montpellier, France. ${ }^{5}$ Department of Plant Systems Biology, VIB, Ghent, Belgium. ${ }^{6}$ Department of Plant Biotechnology and Bioinformatics, Ghent University, Ghent, Belgium. 7Université Lyon 1, Université de Lyon, CNRS, Ecologie Microbienne, UMR 5557, Villeurbanne 69622Cedex, France.

Received: 31 July 2014 Accepted: 19 November 2014 Published online: 10 December 2014

\section{References}

1. Perrine-Walker F, Gherbi H, Imanishi L, Hocher V, Ghodhbane-Gtari F, Lavenus J, Meriem Benabdoun F, Nambiar-Veetil M, Svistoonoff S, Laplaze L: Symbiotic signaling in actinorhizal symbioses. Curr Protein Pept Sci 2011, 12:156-164.

2. Santi C, Bogusz D, Franche C: Biological nitrogen fixation in non-legume plants. Ann Bot 2013, 111:743-767.

3. Pawlowski K, Demchenko KN: The diversity of actinorhizal symbiosis. Protoplasma 2012, 249:967-979.

4. Svistoonoff S, Hocher V, Gherbi H: Actinorhizal root nodule symbioses: what is signalling telling on the origins of nodulation? Curr Opin Plant Biol 2014, 20C:11-18

5. Hocher V, Alloisio N, Auguy F, Fournier P, Doumas P, Pujic P, Gherbi $H$, Queiroux C, Da Silva C, Wincker P, Normand P, Bogusz D: Transcriptomics of actinorhizal symbioses reveals homologs of the whole common symbiotic signaling cascade. Plant Physiol 2011, 156:700-711.

6. Demina IV, Persson T, Santos P, Plaszczyca M, Pawlowski K: Comparison of the nodule vs. root transcriptome of the actinorhizal plant Datisca glomerata: actinorhizal nodules contain a specific class of defensins. PLOS ONE 2013, 8:e72442.

7. Tromas A, Parizot B, Diagne N, Champion A, Hocher V, Cissoko M, Crabos A, Prodjinoto H, Lahouze B, Bogusz D, Laplaze L, Svistoonoff S: Heart of endosymbioses: transcriptomics reveals a conserved genetic program among arbuscular mycorrhizal, actinorhizal and legume-rhizobial symbioses. PLOS ONE 2012, 7:e44742.

8. Oldroyd GED, Harrison MJ, Paszkowski U: Reprogramming plant cells for endosymbiosis. Science 2009, 324:753-754.

9. Schauser L, Roussis A, Stiller J, Stougaard J: A plant regulator controlling development of symbiotic root nodules. Nature 1999, 402:191-195.

10. Heckmann AB, Sandal N, Bek AS, Madsen LH, Jurkiewicz A, Nielsen MW, Tirichine L, Stougaard J: Cytokinin induction of root nodule primordia in Lotus japonicus is regulated by a mechanism operating in the root cortex. Mol Plant-Microbe Interact MPMI 2011, 24:1385-1395.

11. Marsh JF, Rakocevic A, Mitra RM, Brocard L, Sun J, Eschstruth A, Long SR, Schultze M, Ratet P, Oldroyd GED: Medicago truncatula NIN is essential for rhizobial-independent nodule organogenesis induced by autoactive calcium/calmodulin-dependent protein kinase. Plant Physiol 2007, 144:324-335.

12. Borisov AY, Madsen LH, Tsyganov VE, Umehara Y, Voroshilova VA, Batagov AO, Sandal N, Mortensen A, Schauser L, Ellis N, Tikhonovich IA, Stougaard J: The Sym 35 gene required for root nodule development in pea is an ortholog of Nin from Lotus japonicus. Plant Physiol 2003, 131:1009-1017.

13. Soyano T, Kouchi H, Hirota A, Hayashi M: Nodule inception directly targets NF-Y subunit genes to regulate essential processes of root nodule development in Lotus japonicus. PLoS Genet 2013, 9:e1003352.

14. Singh S, Katzer K, Lambert J, Cerri M, Parniske M: CYCLOPS, a DNA-binding transcriptional activator, orchestrates symbiotic root nodule development. Cell Host Microbe 2014, 15:139-152

15. Smit P, Raedts J, Portyanko V, Debellé F, Gough C, Bisseling T, Geurts R: NSP1 of the GRAS protein family is essential for rhizobial Nod factor-induced transcription. Science 2005, 308:1789-1791.

16. Kaló P, Gleason C, Edwards A, Marsh J, Mitra RM, Hirsch S, Jakab J, Sims S, Long SR, Rogers J, Kiss GB, Downie JA, Oldroyd GED: Nodulation signaling in legumes requires NSP2, a member of the GRAS family of transcriptional regulators. Science 2005, 308:1786-1789.

17. Cerri MR, Frances L, Laloum T, Auriac M-C, Niebel A, Oldroyd GED, Barker DG, Fournier J, de Carvalho-Niebel F: Medicago truncatula ERN transcription factors: regulatory interplay with NSP1/NSP2 GRAS factors and expression dynamics throughout rhizobial infection. Plant Physiol 2012, 160:2155-2172.

18. Liu W, Kohlen W, Lillo A, Op den Camp R, Ivanov S, Hartog M, Limpens E, Jamil M, Smaczniak C, Kaufmann K, Yang W-C, Hooiveld GJE, Charnikhova T, Bouwmeester HJ, Bisseling T, Geurts R: Strigolactone biosynthesis in Medicago truncatula and rice requires the symbiotic GRAS-type transcription factors NSP1 and NSP2. Plant Cell 2011, 23:3853-3865.

19. Delaux P-M, Bécard G, Combier J-P: NSP1 is a component of the Myc signaling pathway. New Phytol 2013, 199:59-65.

20. Laloum T, Baudin M, Frances L, Lepage A, Billault-Penneteau B, Cerri MR, Ariel F, Jardinaud M-F, Gamas P, de Carvalho-Niebel F, Niebel A: Two CCAAT-box-binding transcription factors redundantly regulate early steps of the legume-rhizobia endosymbiosis. Plant J Cell Mol Biol 2014, 79:757-768.

21. Sinharoy S, Torres-Jerez I, Bandyopadhyay K, Kereszt A, Pislariu Cl, Nakashima J, Benedito VA, Kondorosi $\mathrm{E}$, Udvardi MK: The $\mathrm{C} 2 \mathrm{H} 2$ transcription factor regulator of symbiosome differentiation represses transcription of the secretory pathway gene VAMP721a and promotes symbiosome development in Medicago truncatula. Plant Cell 2013, 25:3584-3601.

22. Andriankaja A, Boisson-Dernier A, Frances L, Sauviac L, Jauneau A, Barker DG, de Carvalho-Niebel F: AP2-ERF transcription factors mediate Nod factor dependent Mt ENOD11 activation in root hairs via a novel cis-regulatory motif. Plant Cell 2007, 19:2866-2885.

23. Pysh LD, Wysocka-Diller JW, Camilleri C, Bouchez D, Benfey PN: The GRAS gene family in Arabidopsis: sequence characterization and basic expression analysis of the SCARECROW-LIKE genes. Plant J Cell Mol Biol 1999, 18:111-119.

24. Tian C, Wan P, Sun S, Li J, Chen M: Genome-wide analysis of the GRAS gene family in rice and Arabidopsis. Plant Mol Biol 2004, 54:519-532.

25. Laloum T, De Mita S, Gamas P, Baudin M, Niebel A: CCAAT-box binding transcription factors in plants: $Y$ so many? Trends Plant Sci 2013, 18:157-166.

26. Wenkel S, Turck F, Singer K, Gissot L, Le Gourrierec J, Samach A, Coupland G: CONSTANS and the CCAAT box binding complex share a functionally important domain and interact to regulate flowering of Arabidopsis. Plant Cell 2006, 18:2971-2984.

27. Distelfeld A, Tranquilli G, Li C, Yan L, Dubcovsky J: Genetic and molecular characterization of the VRN2 loci in tetraploid wheat. Plant Physiol 2009, 149:245-257.

28. Lévesque-Lemay M, Albani D, Aldcorn D, Hammerlindl J, Keller W, Robert LS: Expression of CCAAT-binding factor antisense transcripts in reproductive tissues affects plant fertility. Plant Cell Rep 2003, 21:804-808.

29. Nakano T, Suzuki K, Fujimura T, Shinshi H: Genome-wide analysis of the ERF gene family in Arabidopsis and rice. Plant Physiol 2006, 140:411-432.

30. Champion A, Hebrard E, Parra B, Bournaud C, Marmey P, Tranchant C, Nicole M: Molecular diversity and gene expression of cotton ERF transcription factors reveal that group IXa members are responsive to jasmonate, ethylene and Xanthomonas. Mol Plant Pathol 2009, 10:471-485.

31. Asamizu E, Shimoda $Y$, Kouchi H, Tabata S, Sato S: A positive regulatory role for LjERF1 in the nodulation process is revealed by systematic analysis of nodule-associated transcription factors of Lotus japonicus. Plant Physiol 2008, 147:2030-2040.

32. Englbrecht $\mathrm{CC}$, Schoof $\mathrm{H}, \mathrm{Böhm} \mathrm{S:} \mathrm{Conservation,} \mathrm{diversification} \mathrm{and}$ expansion of $\mathrm{C} 2 \mathrm{H} 2$ zinc finger proteins in the Arabidopsis thaliana genome. BMC Genomics 2004, 5:39.

33. Fujimoto SY, Ohta M, Usui A, Shinshi H, Ohme-Takagi M: Arabidopsis ethylene-responsive element binding factors act as transcriptional activators or repressors of GCC box-mediated gene expression. Plant Cell 2000, 12:393-404.

34. Ohta M, Matsui K, Hiratsu K, Shinshi H, Ohme-Takagi M: Repression domains of class II ERF transcriptional repressors share an essential motif for active repression. Plant Cell 2001, 13:1959-1968.

35. Frugier F, Poirier S, Satiat-Jeunemaitre B, Kondorosi A, Crespi M: A Krüppel-like zinc finger protein is involved in nitrogen-fixing root nodule organogenesis. Genes Dev 2000, 14:475-482. 
36. Zhang H, Jin J, Tang L, Zhao Y, Gu X, Gao G, Luo J: PlantTFDB 2.0: update and improvement of the comprehensive plant transcription factor database. Nucleic Acids Res 2011, 39(Database issue):D1114-D1117.

37. Oldroyd GED, Downie JA: Coordinating nodule morphogenesis with rhizobial infection in legumes. Annu Rev Plant Biol 2008, 59:519-546.

38. Moreau S, Verdenaud M, Ott T, Letort S, de Billy F, Niebel A, Gouzy J, de Carvalho-Niebel F, Gamas P: Transcription reprogramming during root nodule development in Medicago truncatula. PLOS ONE 2011, 6:e16463.

39. Pré M, Atallah M, Champion A, De Vos M, Pieterse CMJ, Memelink J: The AP2/ERF domain transcription factor ORA59 integrates jasmonic acid and ethylene signals in plant defense. Plant Physiol 2008, 147:1347-1357.

40. Cheng M-C, Liao P-M, Kuo W-W, Lin T-P: The Arabidopsis ETHYLENE RESPONSE FACTOR1 regulates abiotic stress-responsive gene expression by binding to different cis-acting elements in response to different stress signals. Plant Physiol 2013, 162:1566-1582.

41. Eulgem T, Somssich IE: Networks of WRKY transcription factors in defense signaling. Curr Opin Plant Biol 2007, 10:366-371.

42. Kazan K: Negative regulation of defence and stress genes by EAR-motifcontaining repressors. Trends Plant Sci 2006, 11:109-112.

43. Smouni A, Laplaze L, Bogusz D, Guermache F, Auguy F, Duhoux E, Franche C: Research note: the $35 \mathrm{~S}$ promoter is not constitutively expressed in the transgenic tropical actinorhizal tree Casuarina glauca. Funct Plant Bio/ 2002, 29:649-656.

44. Hocher V, Auguy F, Argout X, Laplaze L, Franche C, Bogusz D: Expressed sequence-tag analysis in Casuarina glauca actinorhizal nodule and root. New Phytol 2006, 169:681-688.

45. Alloisio N, Queiroux C, Fournier P, Pujic P, Normand P, Vallenet D, Médigue C, Yamaura M, Kakoi K, Kucho K: The Frankia alni symbiotic transcriptome. Mol Plant-Microbe Interact MPMI 2010, 23:593-607.

46. Edgar RC: MUSCLE: multiple sequence alignment with high accuracy and high throughput. Nucleic Acids Res 2004, 32:1792-1797.

47. Castresana J: Selection of conserved blocks from multiple alignments for their use in phylogenetic analysis. Mol Biol Evol 2000, 17:540-552.

48. Dereeper A, Guignon V, Blanc G, Audic S, Buffet S, Chevenet F, Dufayard J-F, Guindon S, Lefort V, Lescot M, Claverie J-M, Gascuel O: Phylogeny.fr: robust phylogenetic analysis for the non-specialist. Nucleic Acids Res 2008, 36(Web Server issue):W465-W469.

doi:10.1186/s12870-014-0342-z

Cite this article as: Diédhiou et al.: Identification of potential transcriptional regulators of actinorhizal symbioses in Casuarina glauca and Alnus glutinosa. BMC Plant Biology 2014 14:342.

\section{Submit your next manuscript to BioMed Central and take full advantage of:}

- Convenient online submission

- Thorough peer review

- No space constraints or color figure charges

- Immediate publication on acceptance

- Inclusion in PubMed, CAS, Scopus and Google Scholar

- Research which is freely available for redistribution

Submit your manuscript at www.biomedcentral.com/submit
(O) Biomed Central 